\title{
Deciphering the orientation of the aromatic spacer cation in bilayer perovskite solar cells through spectroscopic techniques
}

Meenakshi Pegu, ${ }^{a}$ Samrana Kazim, ${ }^{\text {ac }}$ Thierry Buffeteau, ${ }^{\mathrm{b}}$ Dario M. Bassani, ${ }^{\mathrm{b}}$ and Shahzada $\operatorname{Ahmad}^{\mathrm{a}, \mathrm{c}, *}$

${ }^{a}$ BCMaterials, Basque Center for Materials, Applications, and Nanostructures, UPV/EHU

Science Park, 48940, Leioa, Spain

Email: shahzada.ahmad@bcmaterials.net

${ }^{\mathrm{b}}$ Univ. Bordeaux, CNRS, Bordeaux INP, ISM, UMR 5255, F-33405, Talence, France

${ }^{\mathrm{C}}$ IKERBASQUE, Basque Foundation for Science, Bilbao, 48009, Spain

\begin{abstract}
Slowing the degradation of perovskite-based solar cells (PSCs) is of substantial interest. We engineered the surface by introducing a hydrophobic overlayer on a 3-dimensional perovskite using fluorinated or nonfluorinated aryl ammonium cation spacers. The placement of a fluoroarene cation allows the formation of a bilayer structure i.e., layered/3-dimensional perovskites. By doing so, the surface hydrophobic character increases notably by the virtue of perfluorinated benzene moiety. The fabricated devices thereof gave higher performance and longevity than control devices, in addition to mitigating reliability. The fluoro- phenethylammonium iodide (FPEAI) based devices showed lower non-radiative carrier recombination. To decipher the orientation of the spacer cation in this bilayer structure, we probed the surface by polarization-modulated infrared reflection-absorption spectroscopy and noted substantial differences in the orientation due to the presence of fluorine substitution. We hypothesize that the stronger van der Waals interactions due to the higher electronegativity in FPEAI govern the orientation, performance enhancement, and acts as a barrier to moisture decomposition.
\end{abstract}

Keywords: PM-IRRAS, fluorinated molecules, FPEAI, PEAI, orientation of spacer cation, bilayer perovskite solar cells. 


\section{Introduction}

Hybrid perovskite-based solar cells (PSCs) have witnessed tremendous progress and a rise in performance and reliability. ${ }^{1,2}$ It is known that the photovoltaic (PV) power conversion efficiency (PCE) and reliability are influenced by the quality of the microcrystalline grain, boundary size, and surface properties. ${ }^{3}$ The use of layered perovskites has shown improvement, and efforts have focussed on the employment of interfacial materials to increase reliability. ${ }^{4-6}$ In the case of bilayer perovskites, the crystalline growth condition and orientation of these materials are of paramount importance as they impact interfacial losses and thus PCE. All-inorganic based PSCs display improved stability but also demand higher annealing temperatures, possess relatively lower PCE, and show phase transformation. ${ }^{7}$ Annealing at high temperatures $\left(>250{ }^{\circ} \mathrm{C}\right)$ is not aligned with the inert atmosphere fabrication process such as glove box. Dimensionally reduced perovskites display higher moisture resistance and lower thermal diffusion compared to 3-dimensional (bulk) perovskites. ${ }^{8-10}$ However, the high quantum well, lower quantum and dielectric confinement, and anisotropic charge transport properties ${ }^{11,12}$ make free carriers difficult to generate in pristine layered perovskites, and the high density of acceptor type trap states at the surface limits PCE values. ${ }^{13}$ One approach is to use bilayer formation to increase the light-harvesting properties of the active layers. ${ }^{14}$ Nevertheless, achieving PCE similar to bulk perovskites along with high operational stability remains challenging. ${ }^{15}$ Controlled deposition of a hydrophobic, uniform, and conformal layer on top of a bulk perovskite has been used to mitigate dispersion forces. Arguably, fluorinated compounds with carbon-fluorine (C-F) bonds having low polarizability are a contributing factor to the stability of the material and are deterrence towards moisture. ${ }^{16,17}$ Fluorinated compounds contribute to the formation of hydrogenbonding interactions between electronegative fluorine atoms and the surface of perovskites. ${ }^{19}$ 
The use of partially fluorinated aliphatic amines for the passivation of interfacial defects and fluorous aliphatic spacer groups within layered (fashionably termed 2D) perovskites gave PCE close to $20 \%$, without hysteresis correction and scan speedindependent maximum power point (MPP) data reported. ${ }^{20}$ Similarly, fluorinated tin perovskites were also reported. ${ }^{21}$ Additionally, employing fluoro-halogenated layers as molecular moisture barriers for stability purposes can increase reliability by forming halogen bonding with the bulk perovskites. ${ }^{18,22}$ We reported the use of a perfluoroalkyl-substituted imidazolium derivative as a doping agent compared to iodopentafluorophenol, and the hydrophobic nature of the dopant allows the formation of a protective layer. ${ }^{23} \mathrm{With}$ the surge in the reports dealing with the use of bilayers/passivation/doping agents to improve PCE and moisture resistance, a rapid and effective approach to deduce the molecular orientation of the layer is paramount in deciphering its action. ${ }^{24}$

In addition to imparting moisture stability, the perfluorinated benzene unit possesses electronic properties conducive towards hole extraction while constraining ion migration. Both ion migration and moisture-induced degradation negatively affect the reliability of PSCs, ${ }^{25}$ and an overlayer can intrinsically mitigate such issues. Ion migration, axial rotation, and moisture-induced degradation are interwoven as surface-bound water and ions can diffuse into the absorber layer through grain boundaries to accelerate degradation. ${ }^{26}$

For layered perovskites, in situ growth using bulky organic cations derived from organic salts and the corresponding halides is widely adopted. These react with excess $\mathrm{PbI}_{2}$ present on the surface to form a layered structure post-annealing through the interaction of the $\mathrm{Pb}$-I framework of the bulk perovskite. Placement of a low dimensional perovskite facilitates the nucleation at the interfaces, resulting in minimal structural disorder and higher charge carrier lifetimes. ${ }^{27}$ While fluoroarene bilayer-based PSCs display higher operational stability under humid conditions. ${ }^{17}$ In our efforts to probe interfacial layers that passivate 
surface defects or add functionality, we focus on the role played by surface mitigation and its impact on the stability. Particularly, the non-random orientation of the interfacial layers is decisive in controlling the electronic effects. However, elucidation of a single or molecular layer's organizational order can be extremely challenging, especially when they are placed on hybrid materials due to the inherent complexity of underlying layers. Recently, techniques such as grazing incidence wide-angle X-rays diffraction (GIWAXD), combined nuclear magnetic resonance (NMR), X-ray crystallography, and ab-initio calculations have been used to glean information concerning nature, thickness, and orientation, e.g. PEA spacers in directing the formation of bilayer hybrid perovskites. ${ }^{28,29}$ Considering the plethora of possible interfacial layer compositions, a straightforward technique to probe the composition and organization of such materials would be extremely valuable. Herein, we show that polarization-modulated infrared reflection-absorption spectroscopy (PM-IRRAS) is capable of rapidly providing the necessary information, without using sophisticated techniques and time investment.

PM-IRRAS is a surface-specific IR technique that combines the electric field enhancement induced by IRRAS and the surface selection rules for the study of selfassembled monolayers. ${ }^{30,31}$ The shifting in the energy of the IR transitions can be assigned to the interaction of the various functional groups present with the surface and, similarly to IR spectroscopy, it provides information on a material's composition and organization. It is a non-destructive technique in which band intensities can be used to obtain information on the relative proportion and orientation of different functional groups to the surface. The latter is of significant interest, as it allows one to specifically assign certain orientations to portions of a molecule that may be partially organized on a surface whereas another part of the same molecule is more randomly oriented. To demonstrate the utility of this approach, we have re- 
examined interfacial layers formed by PEAI and FPEAI, which form parallel and perpendicularly oriented monolayers, respectively (Figure 1).

(a)

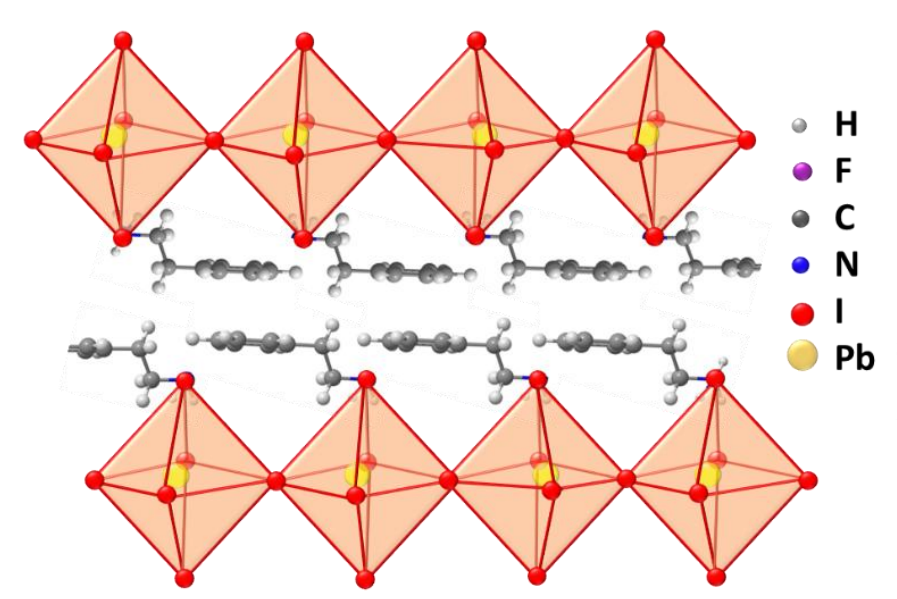

(b) Perpendicular

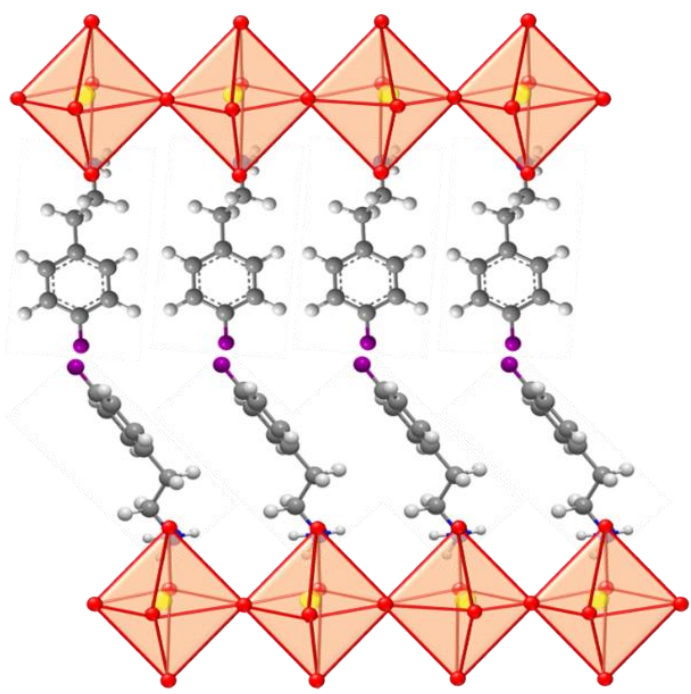

Figure 1. Proposed molecular orientation of (a) PEAI, flat (parallel) orientation, and (b) FPEAI, vertical (perpendicular) orientation.

Our results confirm that both PEAI and FPEAI (Figure S1) are non-randomly ordered on the surface and display different orientations. PEAI lies flat (long axis parallel) on the surface whereas FPEAI is found to be vertically oriented. Moreover, the surface concentration/density of PEAI is constant and is independent of the concentration used for deposition $(0.3$ or $0.7 \%)$, whereas in the case of the FPEAI the surface concentration is higher at $0.7 \%$ than at $0.3 \%$. From this, we may rationalize the advantage of the FPEAI over the non-fluorinated or aliphatic counterpart even though both PEAI and FPEAI improve the crystallinity and moisture tolerance significantly. ${ }^{32}$ We measured a PCE of $20.63 \%$ along with a significant increase in stability than of control and PEAI based PSCs, corroborating that a single $\mathrm{F}$ atom in PEA improves the material's moisture resistance. ${ }^{5}$

\section{Result and Discussions}

\subsection{PM-IRRAS study on bilayer perovskites}


The PM-IRRAS spectra of the pristine perovskites and samples treated with $0.3 \%$ and 0.7\% FPEAI are shown in Figure 2a, along with the isotropic (non-oriented) spectra of FPEAI and the assignment of the principal vibrations. From this we can deduce that the spectral signature of FPEAI is visible in the bilayer perovskites and the intensity of the FPEAI bands increases by a factor of 2.15 which is close to the ratio of concentrations used for deposition $(0.7 \%$ and $0.3 \%)$.

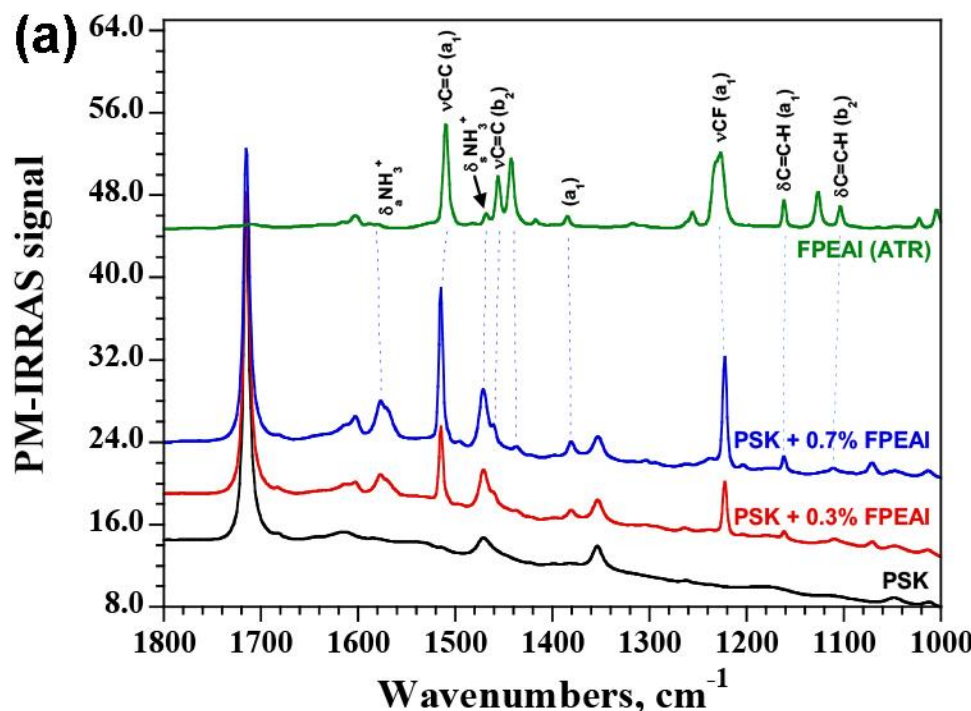

(c)
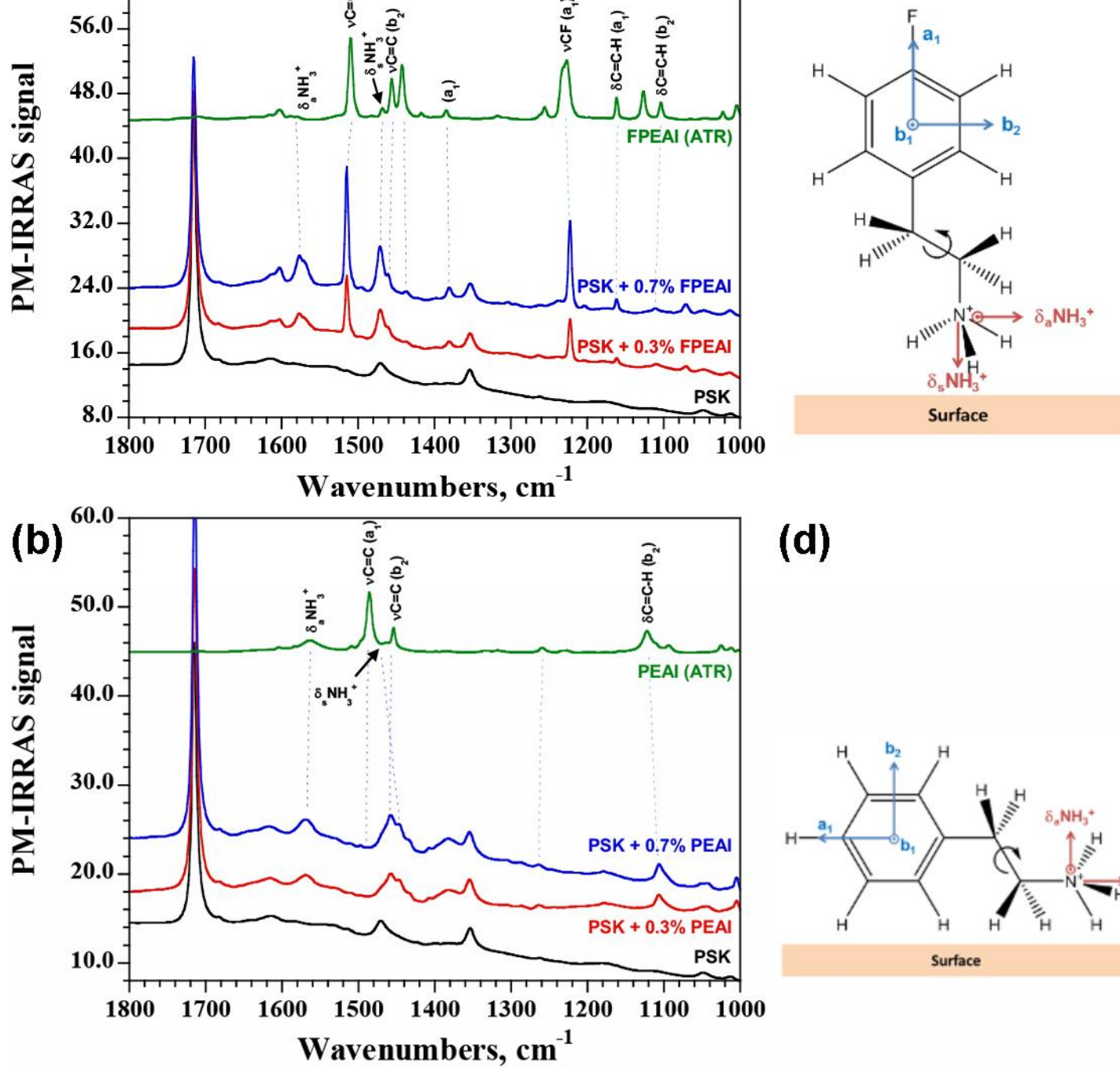

(d)

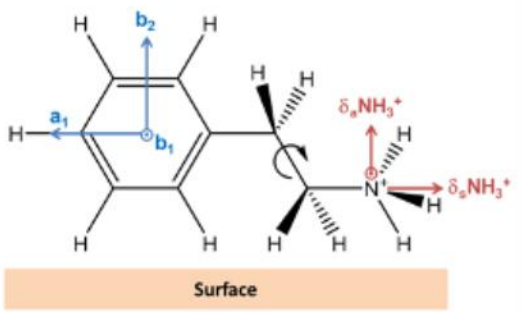

Figure 2. PM-IRRAS spectra of pristine PSK (black), PSK $+0.3 \%$ (red) and $0.7 \%$ (blue) of (a): FPEAI and (b): PEAI. The assignment of the vibrations is from the ATR spectrum (green) of an isotropic sample of the ammonium iodide salt. Proposed orientation of FPEAI (c) and PEAI (d) on the PSK surface in agreement with the enhancement of those transitions 
that are perpendicular to the surface and suppression of the transitions parallel to the surface. The $\mathrm{NH}_{3}$ fragment is disordered and shows no orientational preference.

Concerning the orientation of the molecules on the surface, we note that the bands possessing $a_{l}$ symmetry (aligned with the $\mathrm{C}-\mathrm{F}$ bond) are enhanced, whereas the phenyl ring vibrations possessing $b_{1}$ or $b_{2}$ symmetry (perpendicular to the $\mathrm{C}-\mathrm{F}$ bond) are strongly reduced. From this, we can conclude that the molecule is preferentially oriented with the $\mathrm{C}-\mathrm{F}$ bond perpendicular to the surface (Figure 2c). There is no difference in the intensities of the $\delta_{\mathrm{a}} \mathrm{NH}_{3}{ }^{+}$vibrations to the $\delta_{\mathrm{s}} \mathrm{NH}_{3}{ }^{+}$, suggesting no preferential orientation of the $\mathrm{NH}_{3}{ }^{+}$group. From this information, we conclude that the FPEAI molecules are oriented vertically. The structure is shown with the $\mathrm{NH}_{3}$ oriented towards the surface, but this is arbitrary although in agreement with previous studies. A small shift in the $\mathrm{C}-\mathrm{F}$ vibration is observed, which would be in agreement with the possibility that the fluorine atom may be interacting with the surface.

A similar situation is seen in the PM-IRRAS spectra of PSK treated with $0.3 \%$ and 0.7\% PEAI solutions (Figure 2b). Despite the absence of the easily identifiable C-F bond, the spectral signature of PEAI is nonetheless evident in the treated samples, albeit less intense and somewhat broader than for FPEAI. In the case of PEAI, we note that the band intensities for samples prepared using $0.7 \%$ and $0.3 \%$ solutions are similar (ratio $=1.44$ vs. expected 2.33). In the absence of the $\mathrm{C}-\mathrm{F}$ bond, determining the orientation of the molecule is more challenging. Here, the vibrations of the phenyl ring can be used to provide orientational information. We can see that the bands possessing $a_{1}$ symmetry (aligned with the long axis of the molecule) are decreased in intensity when the molecule is on the surface. This indicates that the molecule is preferentially lying with the long axis parallel to the surface. Additionally, if we compare the intensity of the vibrations with $b$ symmetry, we can deduce 
that the phenyl ring is not flat on the surface, but it is lying edge-on or at an angle since vibrations possessing $b_{2}$ symmetry are visible. Furthermore, no difference in the intensities of the $\delta_{\mathrm{a}} \mathrm{NH}_{3}{ }^{+}$vibrations to the $\delta_{\mathrm{s}} \mathrm{NH}_{3}{ }^{+}$was noted, signifying no preferential orientation of the $\mathrm{NH}_{3}{ }^{+}$group. These observations suggest that the PEAI molecule is oriented horizontally with the phenyl ring perpendicular to the surface (Figure 2d). Remarkably, two bands from the $\delta_{\mathrm{s}} \mathrm{NH}_{3}{ }^{+}$vibration (1446 and $1433 \mathrm{~cm}^{-1}$ ) are present, implying two different environments for the $\mathrm{NH}_{3}$ group. The two different orientations of the molecule may explain the reduced surface coverage observed for PEAI as compared to FPEAI. The vertical orientation of FPEAI occupies a smaller surface area than PEAI which on the contrary lies flat on the surface. Upon increasing the concentration from $0.3 \%$ to $0.7 \%$, the FPEAI molecules can pack vertically on the surface whereas the horizontal surface packing of PEAI is already close to the maximum at $0.3 \%$ concentration.

The UV-visible absorbance spectra of the pristine and of bilayer perovskites (Figure 3a) suggest that varying the percentage of either FPEAI or PEAI has a negligible effect on the perovskite's absorption. (Figure S4). The absorbance profile remains unchanged after three weeks of exposure to the ambient atmosphere. The absorbance spectra of pure-phase layered perovskites $\left(\mathrm{FPEA}_{2} \mathrm{PbI}_{4}, \mathrm{PEA}_{2} \mathrm{PbI}_{4}\right)$ were also measured to identify their optical behavior (Figure S5). 

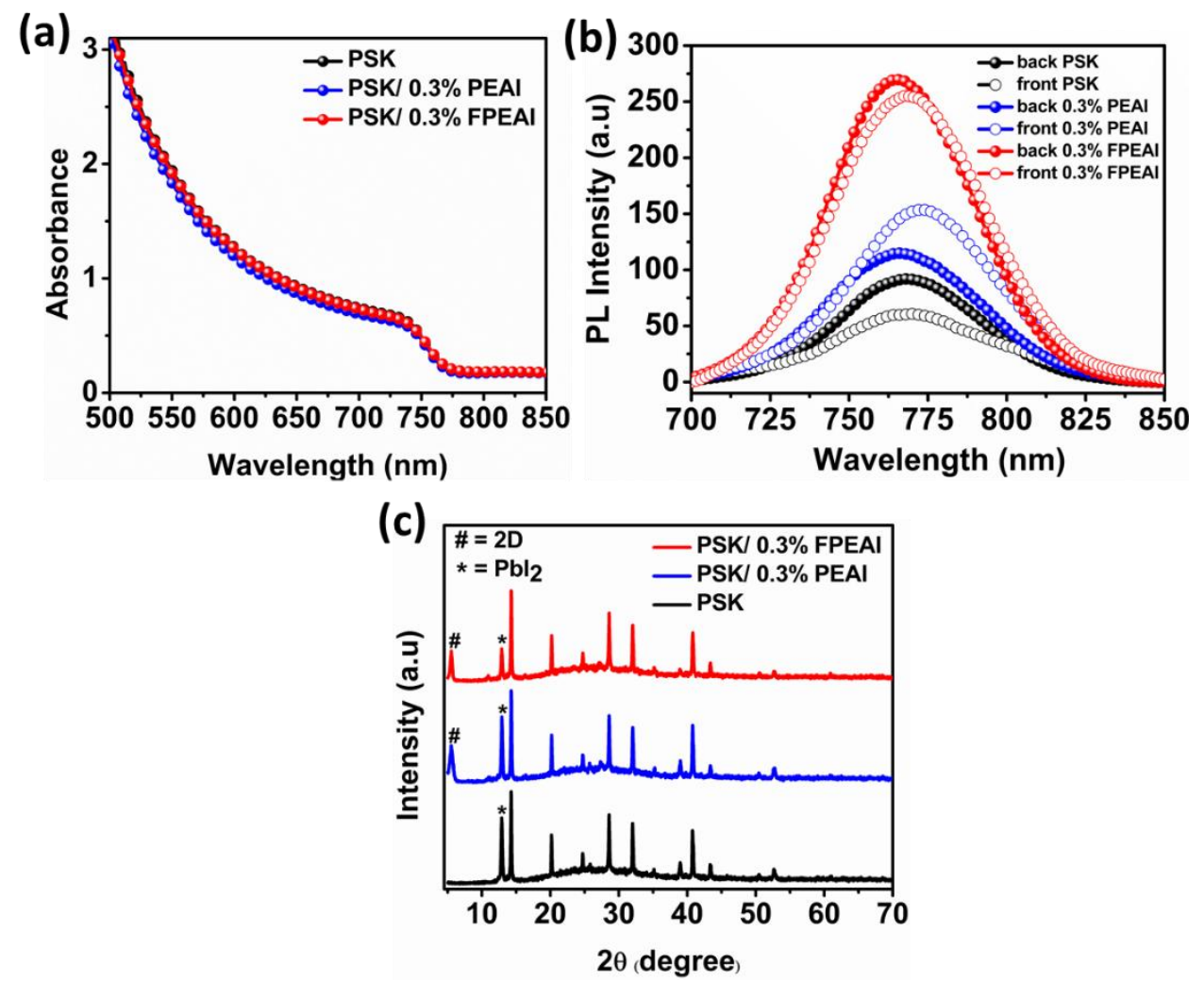

Figure 3. (a) UV-Visible absorption; photoluminescence emission spectra (b) front-side and back-side of the thin films, and (c) X-ray diffraction patterns for pristine PSK and bilayer perovskite.

Steady-state photoluminescence (PL) experiments were made in which the samples were excited either through the glass substrate or directly on the thin film side (Figure $3 b$ ). Quartz substrate without and with $0.3 \%$ PEAI or $0.3 \%$ FPEAI treatment on perovskites was used with an excitation wavelength of $450 \mathrm{~nm}$ having a penetration depth of less than 100 $n m .{ }^{33}$ When the thin film sample was excited from the front side (Figure 3b), this will excite charges that are close to the PEAI or FPEAI/PSK interface, while in case of excitation from the back (glass) side, the beam will strike firstly to the perovskite layer. We noted substantial differences in both side and front illumination shows red shifting in the case of PEAI treated films while backside illumination evidences a blue shift in the case of PEAI and FPEAI treated perovskite films. The small amount of PEAI on the perovskite surface causes a 
redshift of the emission of the perovskite. ${ }^{34}$ The PL emission peak of the bulk PSK is c.a. 768 $\mathrm{nm}$ and $770 \mathrm{~nm}$ when excited from the quartz (back) and front side respectively, which is consistent with the absorption results. The small shift in the peak position of bulk PSK might be due to the variation in crystal size and microstructure on the top and bottom of the surface. $^{35}$ Upon formation of low-dimensional perovskite layers due to PEAI/FPEAI treatment, the PL intensity increases irrespective of the illumination side. We also noted a stronger PL intensity that is blue-shifted $(2-3 \mathrm{~nm})$ for FPEAI treated films (irrespective of excitation side). The observation of strong PL intensity in the case of FPEAI treated perovskite regardless of the illumination side can be ascribed to the filling of traps when less concentrated FPEAI was used which diffuses inside the grain along with the grain boundaries and the surface during the annealing process.

X-ray diffraction analyses were performed to elucidate changes in the structural properties (Figure 3c) for pristine and $0.3 \%$ treated bilayer perovskites. The PEAI and FPEAI dissolved in isopropanol (IPA) were coated on the bulk (3D) perovskite layer and annealed at $100^{\circ} \mathrm{C}$ to allow in situ growth for the formation of a bilayer perovskite. The diffractograms are unchanged and this suggests that the over layers of the organic spacer group on top of bulk perovskites do not substantially alter the structure. The peak at $12.8^{\circ}$ is ascribed to the excess $\mathrm{PbI}_{2}$ phase present in the films. The presence of well-defined layered perovskite is reflected from the characteristic peak patterns at lower angle $2 \theta$ region $\left(<10^{\circ}\right)$, which are associated with the formation of a bilayer perovskite. The increase in signal of the perovskite peak at $14.33^{\circ}$ indicates higher crystallinity and large grain size of the films. The X-ray diffraction pattern with $0.5 \%$ and $0.7 \%$ treated bilayer perovskite (Figure S6), reflects the high-intensity peaks of layered perovskite with increment in the concentration of the bulk layer. 
To probe the molecular interactions between the bulk perovskite and the bulky organic cations, we collected FTIR spectra for the corresponding pure phase layered perovskite, bulk, and the bilayer perovskite layers (Figure S7) ${ }^{20}$ A similar profile was noted in all the samples (pristine and $0.3 \%$ PEAI, FPEAI treated) in the higher wavenumber region $(3000-3500 \mathrm{~cm}$ ${ }^{1}$ ), which corresponds to the $\mathrm{N}-\mathrm{H}$ stretching frequency of the formamidinium cation (FA) cations.
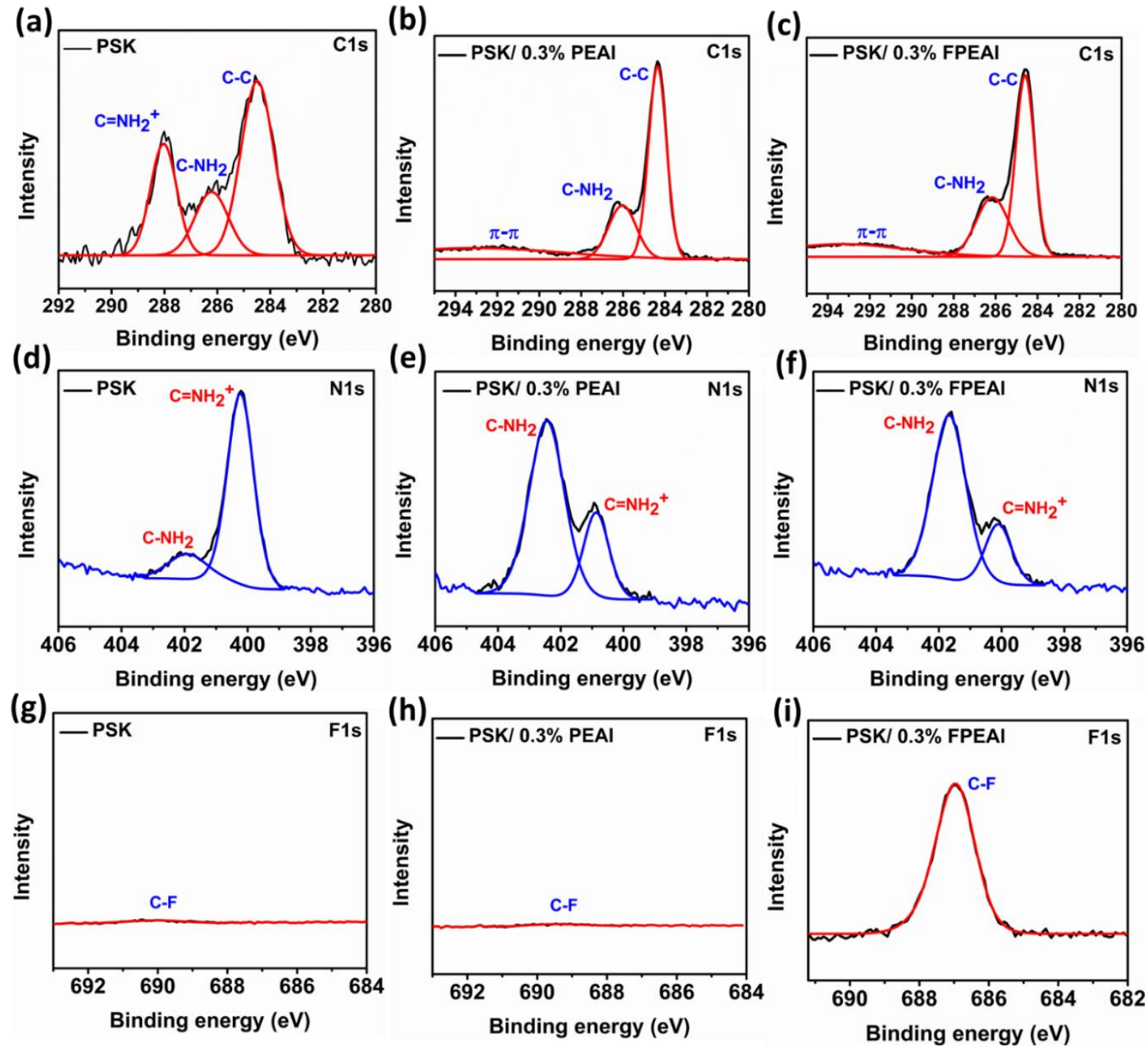

Figure 4. X-ray photoelectron spectroscopy (XPS), (a-c) high-resolution deconvoluted spectra of C1s, (d-f) N 1s peaks, and (g-i) F 1s peaks of the pristine and bilayer perovskite. 
To investigate the elemental distribution on the surface, we performed X-ray photoelectron spectroscopy (XPS) of thin films. The core-level peaks of C, N, and F (Figure 4) suggest the formation of bilayer perovskite, while the strong $\mathrm{C} 1 \mathrm{~s}$ spectra in the profile are attributed to the ex-situ measurements condition (Figure 4a-c). In the case of bulk perovskite, the presence of MA and FA components from the C1s and N1s spectra is supported by the presence of peaks at $286.21 \mathrm{eV}$ and $288.03 \mathrm{eV}$. While the N1s peaks of MA and FA with $400.24 \mathrm{eV}$ and $401.9 \mathrm{eV}$ positions are per report. Layered perovskites with PEAI and FPEAI display a characteristic peak at the binding energy of $292.03 \mathrm{eV}$ due to the presence of the aromatic (phenyl) group in the $\mathrm{PEA}^{+}$or $\mathrm{FPEA}^{+}$cations. The peaks at $402.44 \mathrm{eV}$ and 401.68 $\mathrm{eV}$ of the N1s spectra (Figure 4d-f) are assigned to the C-N of the amino groups in the cations (PEAI, FPEAI). The fluoro substitution at the phenyl group $\left(\mathrm{FPEA}^{+}\right)$was confirmed by the appearance of a $686.97 \mathrm{eV}$ peak in the $\mathrm{F} 1 \mathrm{~s}$ spectrum (Figure $4 \mathrm{~g}-\mathrm{i}$ ). The $\mathrm{Pb} 4 \mathrm{f}$ and $\mathrm{I} 3 \mathrm{~d}$ peaks of the bulk as well as the layered perovskite (Figure S8) shows the different binding energies of the organic moieties on the surface of the bulk perovskite.

The microstructure of the bulk and bilayer perovskites was studied with the help of scanning electron microscopy (Figure 5b-d). The images suggest films composed of a uniform distribution of crystals without any voids. A significant difference for the bilayer perovskites was the presence of larger sized crystals, which in turn minimizes the number of grain boundaries. We note from the images that increasing the thickness (concentration) of PEAI or FPEAI has a small impact on the shape of the microstructure, while orderly crystal size with the coarser surface was also noted (Figure S9). The average grain size of pristine perovskites was $300 \mathrm{~nm}$, while for PEAI or FPEAI we find it to be around $550 \mathrm{~nm}$. 

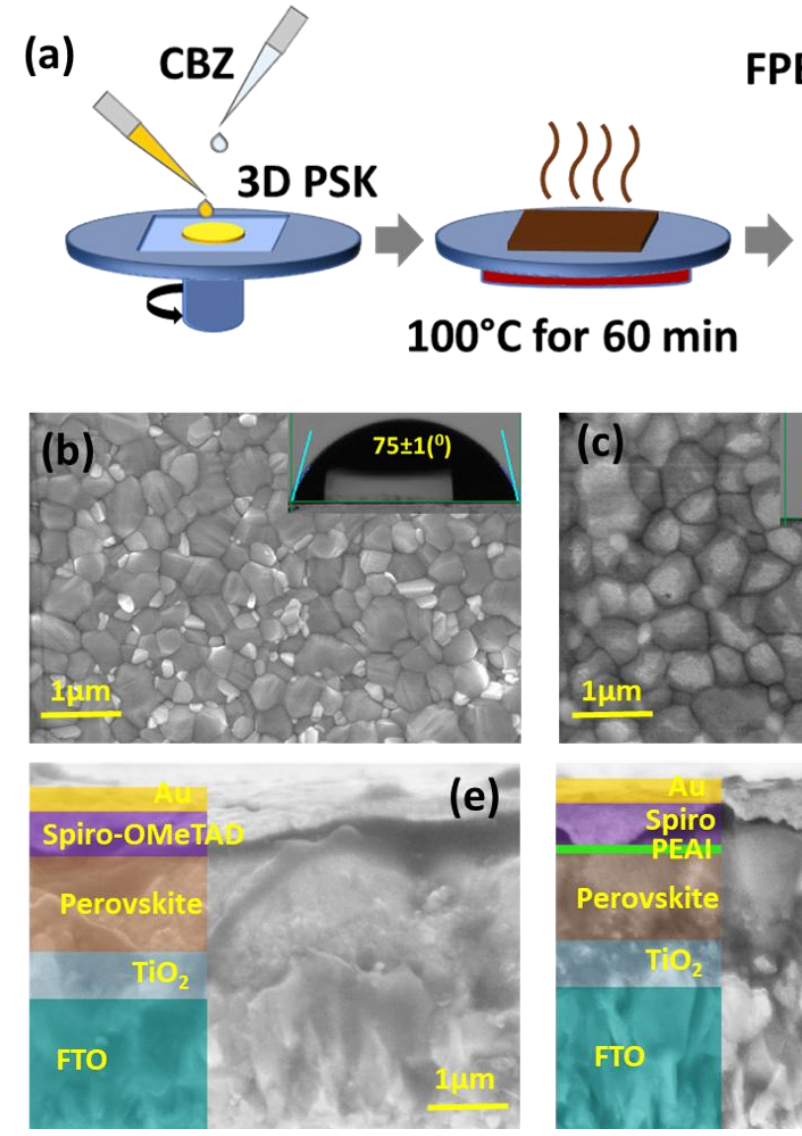
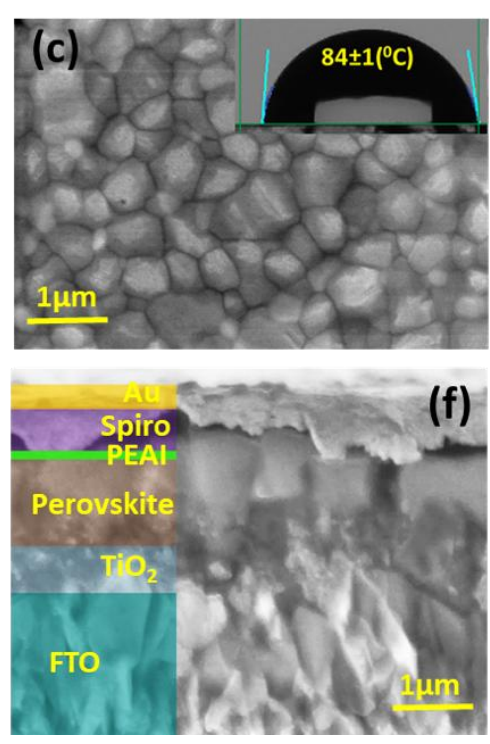

FPEI/PEAI

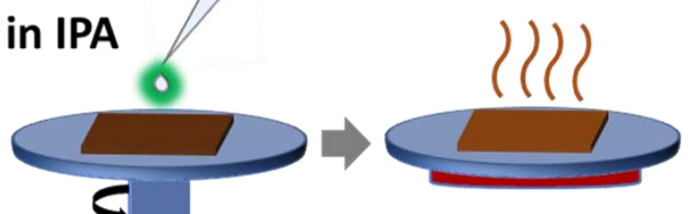

$100^{\circ} \mathrm{C}$ for $8 \mathrm{~min}$

Figure 5. (a) Schematic illustration of bilayer perovskite formation; microstructures and contact angle measurements for (b) pristine perovskite, (c) with $0.3 \%$ PEAI, d) with $0.3 \%$ FPEAI; Cross sectional SEM images of PSCs with (e) pristine perovskite, (f) with $0.3 \%$ PEAI and $(\mathrm{g})$ with $0.3 \%$ FPEAI.

We undertook goniometer contact angle measurements to probe the hydrophobicity induced by the deposition of the PEAI or FPEAI layers. Pristine perovskites showed an average value of $74^{\circ}$, while the bilayer perovskites layered with PEAI gave an average value of $84^{\circ}$. This value increased significantly with FPEAI coated perovskites to give a contact angle value of $96^{\circ}$. This signals the hydrophobic character induced on the surface (Figure 5bd) owing to the presence of fluorinated molecules. The layer thickness was approximated by performing cross-sectional SEM imaging experiments (Fig. 5e-g) according to our experimental protocol. The cross-section SEM images represent a defined layer-by-layer 
structure with intimate interfaces, reflecting the architect adopted. The mixed perovskite $\left(\mathrm{FA}_{0.15} \mathrm{MA}_{0.85} \mathrm{PbI}_{3-\mathrm{x}} \mathrm{Br}_{\mathrm{x}}\right)$ layer represents the thickness of $(t \mathrm{u} \sim 453 \mathrm{~nm})$, while PEAI and FPEAI showed a thickness which we speculate to be $<20 \mathrm{~nm}$, could be hardly measured as it lies as a thin sandwich layer between the 3D perovskite and the hole transport layer (Figure $5 \mathrm{e}-\mathrm{g})$.

Though the performance was not the main aim of the current investigation, we fabricated PSCs to report figures of merit with an objective for added optimization. PSC with an $\mathrm{FTO} / b \& m p-\mathrm{TiO}_{2} / 3 \mathrm{D}$-/layered perovskites/Spiro-OMeTAD/Au architecture (Figure 6a) were fabricated and the corresponding band diagram (Figure 6b) suggests proper alignment for the transport of charges. The device $J$ - $V$ curves (Figure $6 \mathrm{c}$ ) and the derived PV values are tabulated in Table 1. Pristine perovskites-based PSCs yielded a PCE of $17.55 \%$, with a $V_{\text {oc }}$ of $1072 \mathrm{mV}, J_{\mathrm{sc}}$ of $22.96 \mathrm{~mA} \mathrm{~cm}{ }^{-2}$, and FF of $71.27 \%$, while the device fabricated with bilayer perovskites showed improved performance. The PSC fabricated with PEAI showed significantly boosted $V_{\mathrm{oc}}$ of $1087 \mathrm{mV}, J_{\mathrm{sc}}$ of $25.01 \mathrm{~mA} \mathrm{~cm} \mathrm{c}^{-2}$, and $\mathrm{FF}$ of $71.85 \%$, with an overall PCE of $19.12 \%$, while in the case of FPEAI based bilayer perovskites a PCE of $20.63 \%$ was measured, with a $V_{\text {oc }}$ of $1107 \mathrm{mV}$, improved $J_{\mathrm{sc}}$ and $F F$ value of $25.06 \mathrm{~mA} \mathrm{~cm}{ }^{-2}$, 74.37\% respectively. The use of PEAI/FPEAI also passivate the surface defects and lower the non-radiative charge recombination and boosted the photovoltage by $40 \mathrm{mV}$. The orientation of molecules (PEAI/FPEAI) is fundamental to the device performance and FPEAI displays preferred orientation which promotes an interlayer formation that can more effectively extract and transport charge carriers to yield higher $F F$. The $F F$ in FPEAI based bilayer perovskite was significantly higher than bulk and PEAI based bilayer perovskites, suggesting the perpendicular orientation of the overlayer is decisive to transport charge effectively. The statistical data of fifteen devices for the pristine as well as the bilayer perovskite-based PSCs 
are summarized (Figure S10). The integrated current density values derived from the incident photon-to-current conversion efficiency (IPCE) follow the values obtained from $J$ - $V$ curves (Figure $6 \mathrm{~d}$ and S12). In the wavelength region of $400-600 \mathrm{~nm}$, over $85 \%$ photon-toelectron conversion was noted and FPEAI-based PSC showed higher response with a shoulder shift to the red part of the spectrum (extended absorption). The molecular stacking of the organic cations attached to the perovskite layer may promote interlayer electronic coupling that in turn allows better charge transfer in the PSCs. The out-of-plane conductivity in FPEA to the molecular stacking with both the organic and inorganic phases was deduced to be higher and can influence the current density of the PSCs. High charge carrier conduction and improved electrical conductivity with the FPEAI treatment were reported and high current density in the fabricated PSCs stems from this. Further, higher light absorption by the PEAI/FPEAI treated perovskite, as reflected by the PL spectra, also supports photocurrent value.

The hysteresis index (HI) was calculated for the PSCs by measuring $J$ - $V$ curves under the forward and reverse scan directions (Table 1). The bilayer perovskites-based PSCs showed a lower HI value of 0.058 for PEAI and 0.061 for FPEAI treated PSCs as compared to pristine perovskites which were 0.144 (Figure S11). 
(a)

(c)
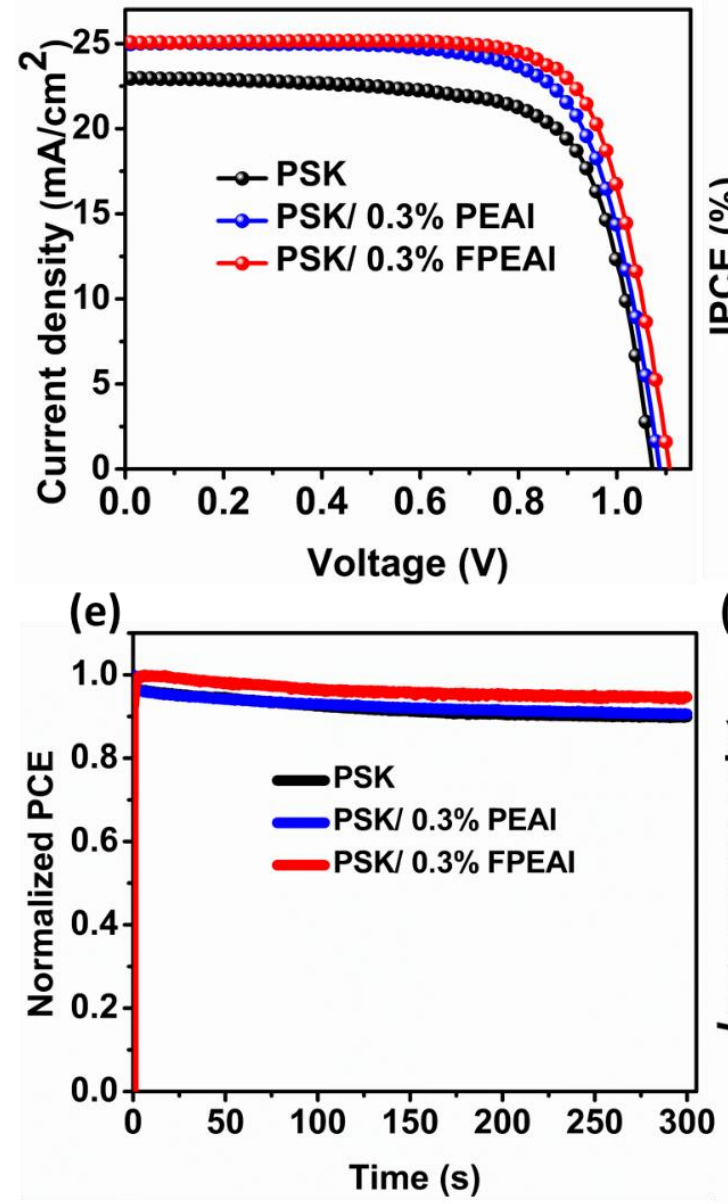

(b)

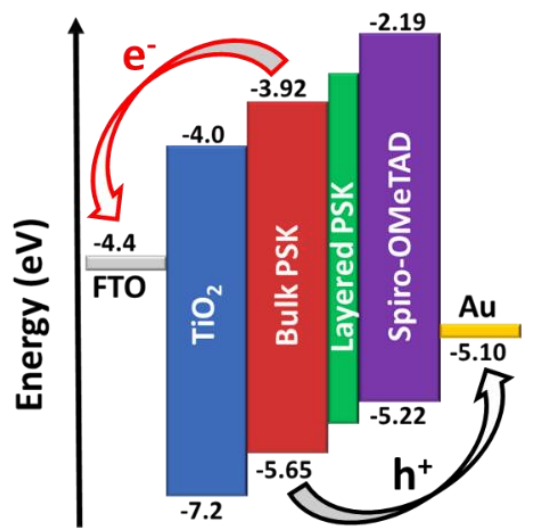

(d)

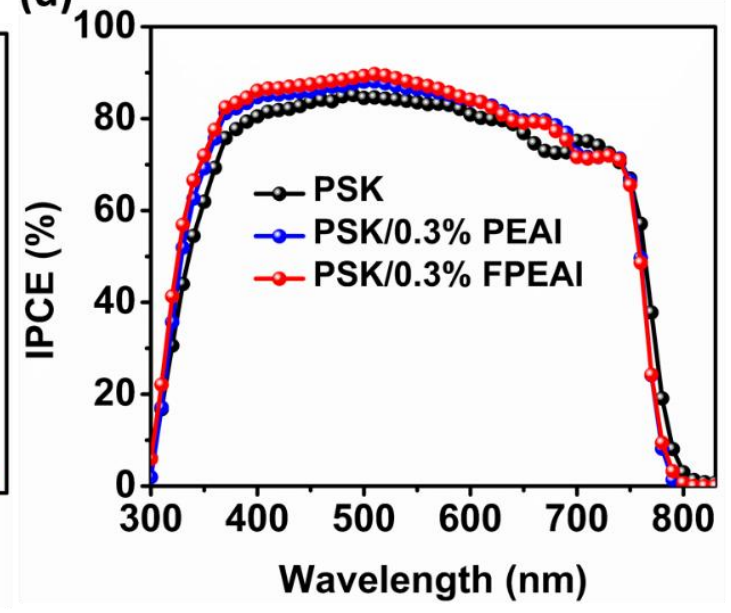

(f)

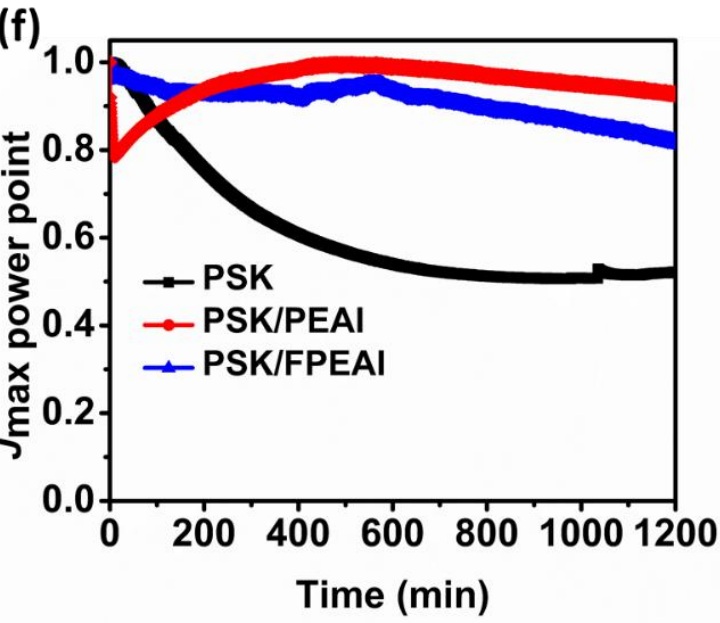

Figure 6. (a) Device architecture of the fabricated PSCs, (b) band diagram of the fabricated PSC, (c) current-voltage characteristics of PSCs based on pristine and bilayer perovskites with $0.3 \%$ PEAI and $0.3 \%$ FPEAI under Air-Mass (AM) $1.5 \mathrm{G}$ illumination, (d) corresponding EQE, (e) steady-state output for $300 \mathrm{~s}$ at $0.8 \mathrm{~V}$, and (f) maximum power point tracking for the pristine and bilayer perovskite with $0.3 \%$ PEAI and $0.3 \%$ FPEAI at $0.8 \mathrm{~V}$ without encapsulation for 1200 mins under 1 sun illumination at ambient condition. 
Table 1: Photovoltaic parameters for bulk and bilayer fabricated perovskites solar cells.

\begin{tabular}{llcclll}
\hline Samples & $\begin{array}{l}\boldsymbol{V}_{\text {oc }} \\
(\mathbf{m V})\end{array}$ & $\boldsymbol{J}_{\mathbf{s c}}\left(\mathbf{m A} / \mathbf{c m}^{2}\right)$ & FF (\%) & $\begin{array}{l}\text { PCE } \\
(\%)\end{array}$ & $\begin{array}{l}\text { Avg. } \\
\mathbf{P C E}\end{array}$ & HI \\
\hline PSK & 1072 & 22.96 & 71.27 & 17.55 & 16.87 & 0.144 \\
\hline $\begin{array}{l}\text { PSK/0.3\% } \\
\text { PEAI }\end{array}$ & 1087 & 25.01 & 71.85 & 19.54 & 18.85 & 0.058 \\
\hline
\end{tabular}

\begin{tabular}{lllllll}
\hline PSK/0.3\% & 1107 & 25.06 & 74.37 & 20.63 & 19.25 & 0.061
\end{tabular}
FPEAI

perovskites based on FPEAI therefore gave higher PCE than PEAI based PSCs. To determine the influence of layered structure on the operational stability of PSCs, we performed maximum power point tracking (MPPT) of the un-encapsulated devices under ambient condition and at 1 sun illumination (Figure 6 e,f). The pristine perovskite showed lower performance as compared to PEAI based layered PSCs, while the PSCs based on FPEAI showed linear response and negligible loss in performance. Apart from improving the PCE, steady output performance is fundamental to evaluate the performance of bilayer PSCs. The bilayer PSCs was also probed for long-term stability to acquire the self-life period, where the un-encapsulated devices were kept in a dry box and measured on a regular interval.

The electrical parameters such as built-in-potential $\left(V_{b i}\right)$, and depletion layer width $(W)$ were deciphered by using Mott-Schottky analysis (Figure 7a) under dark conditions. The Mott-Schottky plot for the fabricated PSCs yields a straight region from which $V_{b i}$ was derived from the intercept on the bias axis. ${ }^{36} V_{b i}$ for the bilayer perovskites-based PSC increases to $1003 \mathrm{mV}(0.3 \%$ PEAI treatment $)$ and $1050 \mathrm{mV}(0.3 \%$ FPEAI treatment $)$ as compared to $950 \mathrm{mV}$ for the pristine PSCs without the interfacial layer. Subsequently, the depletion width $\left(W=\sqrt{\frac{2 \varepsilon \varepsilon_{0} V_{b i}}{q N}}\right)$ increases from $99.98 \mathrm{~nm}$ (pristine) to $106.13 \mathrm{~nm}(0.3 \%$ FPEAI) for the bilayer PSCs. To elucidate the device kinetics that led to the increment in PCE with bilayer PSCs, we probed the charge accumulation behavior by examining the Nyquist plots through electrical impedance spectroscopy (EIS). The recombination resistance 
(Figure 7c), and charge transfer resistance (Figure 7d) as function of voltage was were derived from Nyquist plots (Figure 7b). A single arc was displayed, a characteristic of mixed perovskites and the equivalent circuit used is shown in Figure S13. ${ }^{37,38} \mathrm{We}$ can deduce from the curves that the recombination resistance follows a similar trend and at voltage close to $V_{\text {oc }}$, they display lower recombination, signaling the main contribution of recombination processes are in the bulk of the perovskite material not at the interface. Further, the use of bilayer perovskites irrespective of PEAI or FPEAI shows lower recombination resistance, suggesting its role in suppressing non-radiative recombination losses. Recombination resistance, near to the open-circuit voltage, is higher for bilayer perovskite-based PSCs, signaling a reduction in the non-radiative recombination defects. Bulk perovskites-based PSCs showed a trap-state recombination, while a change in the slope was noted for bilayer perovskites. Similarly, the charge transfer resistance was lower for bilayer perovskites based PSCs, illustrating its advantageous properties stemmed from filling of grain boundaries as well as defects passivation. 
(a)
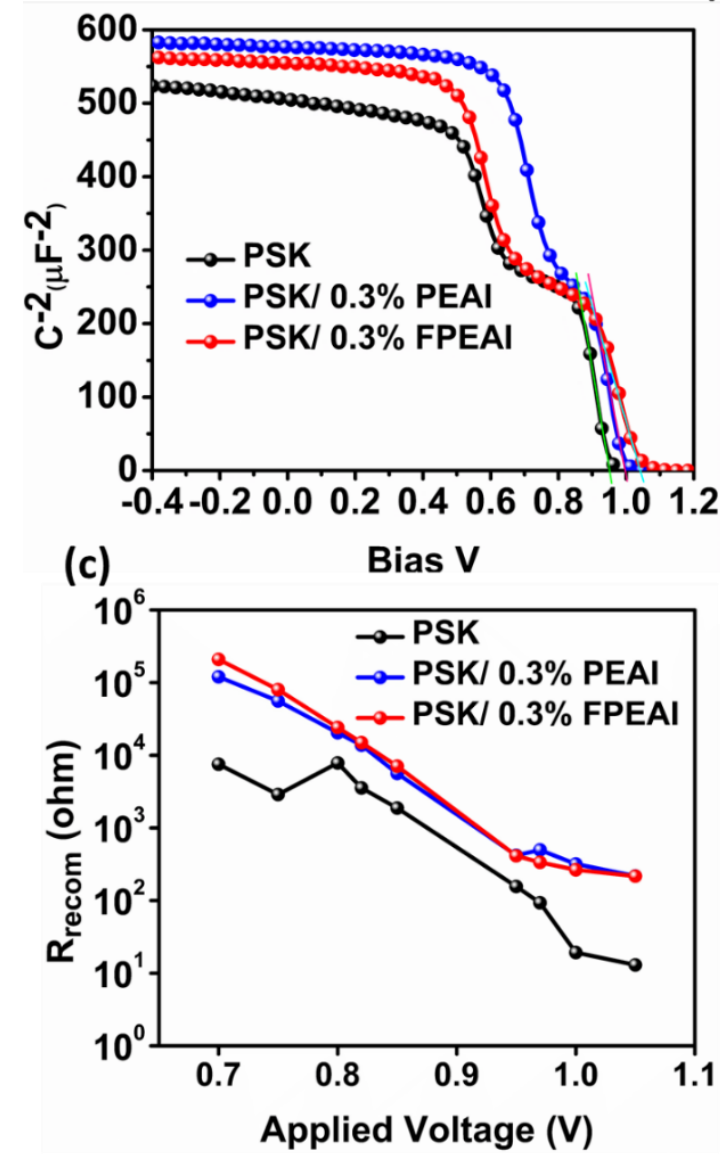

(b)

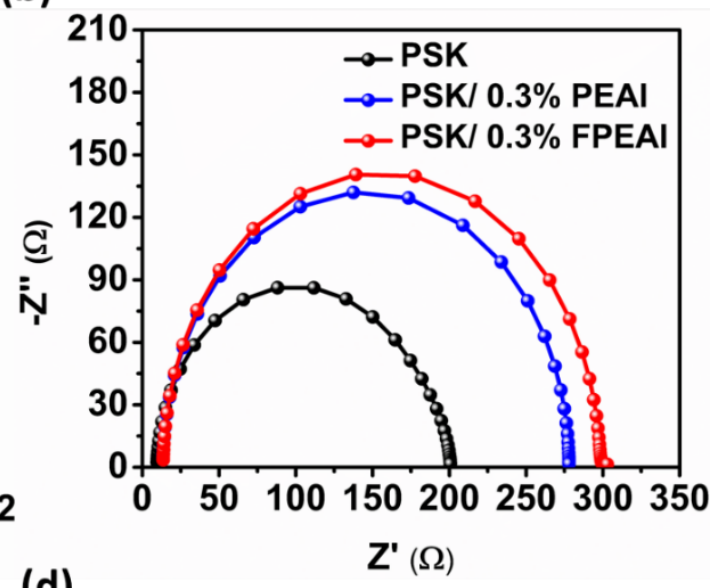

(d)

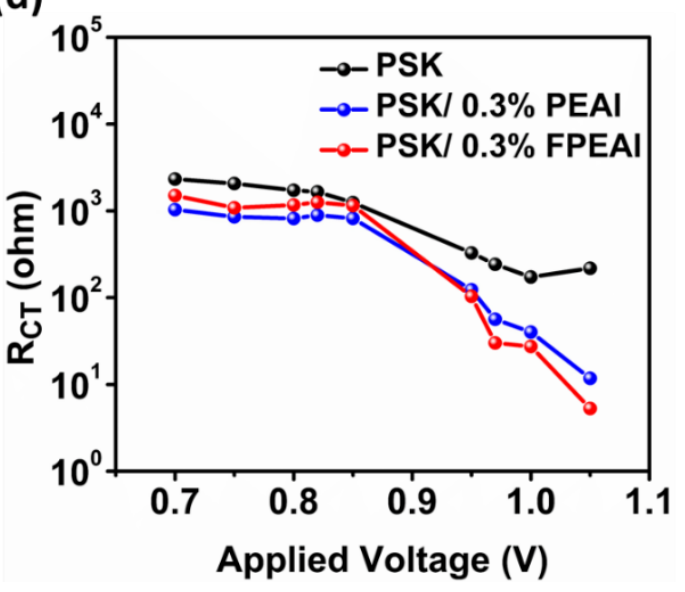

Figure 7. (a) Mott-Schottky plot for the pristine and bilayer perovskites with $0.3 \%$ PEAI and 0.3\% FPEAI, (b) Nyquist plot for the pristine and bilayer perovskites $0.3 \%$ PEAI and $0.3 \%$ FPEAI, (c) recombination resistance at the applied voltage for pristine and bilayer perovskites $0.3 \%$ PEAI and $0.3 \%$ FPEAI, and (d) charge transfer resistance at the applied voltage for pristine and bilayer perovskites $0.3 \%$ PEAI and $0.3 \%$ FPEAI.

\section{Conclusion}

We report inclusive methodology to fabricate a bilayer (layered/bulk) perovskite-based solar cells which combine the panchromatic absorption characteristics of bulk perovskites with the stability of the layered structure. This in turn increases the stability of the perovskite structure while reducing the non-radiative charge recombination through suppression of defects. By employing PM-IRRAS studies we decipher the orientation of PEAI and FPEAI, which are 
located with the phenyl groups parallel and perpendicular to the interface, respecitvely. Due to its high electro-negativity, strong van der Waals interactions, and surface tension, FPEAI layers perpendicular to the surface while PEAI lies parallel to the bulk surface. We further evidence the decisive role of perpendicular orientation for effective charge transport and extraction. Bilayer formation may also stabilize the surface through hydrogen bonding contributions. Such synergistic approaches gave improve PV performance and the device's efficiencies by over $20 \%$.

\section{Experimental Section:}

\section{Materials}

Lead Iodide (99.9\%) and formamidinium iodide (FAI) were purchased from Tokyo Chemical Industry (TCI) and employed as received. Lead bromide was procured from Sigma-Aldrich. 2-(4-Fluorophenyl)ethylammonium iodide (FPEAI), phenethylammonium iodide (PEAI), methylammonium bromide (MABr) were purchased from Dyesol. Common solvents such as DMF, DMSO, isopropanol, ethanol, chlorobenzene were purchased from Acros Chemicals and used as received.

\section{Device Fabrication}

The fluorine-doped tin oxide (FTO) coated glass (TEC15) was used as substrate and was cleaned in sequence before use. Ultrasonication (2\% Hellmanex water solution for 30 minutes) followed by rinsing with deionized water, acetone, and then isopropanol (IPA) was used and finally, the substrates were treated under UV-Ozone for 30 minutes for the removal of organic residue on the surface. $\mathrm{TiO}_{2}$ compact layer was deposited on this substrate by spray pyrolysis at $500{ }^{\circ} \mathrm{C}$ using a precursor solution of titanium diisopropoxide bis(acetylacetonate) in anhydrous ethanol (1:19), and the substrates were annealed at $500{ }^{\circ} \mathrm{C}$ for further 30 minutes and left to cool down. The mesoporous $\mathrm{TiO}_{2}$ layer was deposited on top of this through spin coating process $\left(30 \mathrm{~s}\right.$ at $4000 \mathrm{rpm}$ ), for this, a $\mathrm{TiO}_{2}$ paste (Dyesol 30 
NR-D) was diluted to $1: 8$ in ethanol. The $\mathrm{TiO}_{2}$ coated substrates were annealed first at 125 ${ }^{\circ} \mathrm{C}$, followed by $500{ }^{\circ} \mathrm{C}$ using a programmable ramp in four-step heating, and were finally maintained at $500^{\circ} \mathrm{C}$ for $30 \mathrm{~min}$ to acquire anatase phase. On attaining room temperature, the substrates were treated under UV-Ozone for 30 minutes and transferred to an argon-filled glove-box for perovskite layer deposition. The precursor solution for preparing the mixed perovskite $\left(\mathrm{MAPbBr}_{3}\right)_{0.15}\left(\mathrm{FAPbI}_{3}\right)_{0.85}$ was prepared by dissolving FAI $(1 \mathrm{M}), \mathrm{PbI}_{2}(1.2 \mathrm{M})$, $\operatorname{MABr}(0.2 \mathrm{M})$, and $\mathrm{PbBr}_{2}(0.2 \mathrm{M})$ in anhydrous DMF: DMSO 4:1 (v:v). The perovskite solution was spin-coated in a two-step sequence at 1000 and $6000 \mathrm{rpm}$ for 10 and $30 \mathrm{~s}$, respectively. During the second step, $110 \mu \mathrm{L}$ of chlorobenzene was dripped as an antisolvent approach on the substrate $5 \mathrm{~s}$ before the end of the spinning process. The perovskite-coated substrates were annealed at $100{ }^{\circ} \mathrm{C}$ for $1 \mathrm{~h}$ in a glovebox. On acquiring room temperature, the layered perovskites $(0.3 \%, 0.5 \%$, and $0.7 \%$ PEAI /FPEAI solution in isopropanol) were deposited and annealed at $100{ }^{\circ} \mathrm{C}$ for 8 minutes. Once it acquires room temperature, SpiroOMeTAD (70 $\mathrm{mM})$ as hole selective layer was deposited by dissolving in chlorobenzene. Spiro-OMeTAD was doped by adding bis-(trifluoromethylsulfonyl)imide lithium salt (LiTFSI) and 4-tert-Butylpyridine ( $t$-BP) in the molar ratio 0.5 and 3.3 respectively. $40 \mu \mathrm{L}$ of HTM solutions were spin-coated atop of the perovskite layer at $4000 \mathrm{rpm}$ for $30 \mathrm{~s}$, in an argon-filled glove box. The device fabrication was finished by evaporating an Au layer of 70 $\mathrm{nm}$ as a cathode under a low vacuum $\left(10^{-6}\right.$ torr). All solutions were prepared inside an argonfilled glove box with controlled moisture and oxygen conditions $\left(\mathrm{O}_{2}<10 \mathrm{ppm}, \mathrm{H}_{2} \mathrm{O}<2 \mathrm{ppm}\right)$.

\section{Device characterization}

The device photovoltaics parameters were measured utilizing current density-voltage $(J-V)$ curves, registered with a Keithley 2400 source-meter under AM $1.5 \mathrm{G}, 100 \mathrm{~mW} \mathrm{~cm}{ }^{2}$ illumination from a $450 \mathrm{~W}$ AAA solar simulator (ORIEL, 94023 A). NREL certified monocrystalline silicon solar cell was used for calibration. A black metal mask $\left(0.1 \mathrm{~cm}^{2}\right)$ was 
used over the square size active area $\left(0.5 \mathrm{~cm}^{2}\right)$ to reduce the influence of scattered light. The devices were measured at a scan rate: $100 \mathrm{mV} \mathrm{s}^{-1}$, pre-sweep delay: $10 \mathrm{~s}$ ). The external quantum efficiency (EQE) measurements were carried out using a 150W Xenon lamp attached to with Bentham PVE300 motorized 1/4m monochromator as the light source.

\section{Thin-film Characterization}

For optical characterization, thin films were prepared on quartz substrate by spin coating of $\left(\mathrm{MAPbBr}_{3}\right)_{0.15}\left(\mathrm{FAPbI}_{3}\right)_{0.85}$ with $(0.3 \%, 0.5 \%, 0.7 \%)$ concentration of PEAI or FPEAI / PSK was used. X-ray diffractograms were recorded using the D8 Advance diffractometer from Bruker (Bragg-Bretanto geometry, with an X-ray tube $\mathrm{Cu} \mathrm{K \alpha}, \lambda=1.5406$ angstrom). A scan of $5-10^{\circ}$ was selected with an acquisition time of $1 \%$ min. The absorption spectra were measured under a UV-Vis-IR spectrophotometer (Varian Cary 50 UV/Vis Spectrophotometer). Photoluminescence (PL) steady-state measurements were recorded with a spectrophotometer (Perkin Elmer Instrument LS55). XPS measurements were carried out on a SPECS system (Berlin, Germany) equipped with Phoibos 150 ID-DLD analyzer with monochromator A1 Ka radiation $(1486.7 \mathrm{eV})$.

PM-IRRAS spectra of the mixed perovskites spin-coated onto gold substrates $(15 \times 20 \mathrm{~mm})$, before and after different PEAI and FPEAI treatment $(0.3$ and $0.7 \%)$, were recorded on a Thermo Nicolet Nexus 670 FTIR spectrometer at a resolution of $4 \mathrm{~cm}^{-1}$ during $4 \mathrm{~h}$ acquisition time. All spectra were collected in a controlled dry environment $(\mathrm{RH}=3 \%)$ protected from ambient light. Experiments were performed at an incidence angle of $75^{\circ}$ using an external homemade goniometer reflection attachment and adding a $\mathrm{ZnSe}$ photoelastic modulator (PEM, Hinds Instruments, type III) after the polarizer. The ATR spectrum of the treated perovskites was recorded on the same FTIR spectrometer using a Silver-Gate (germanium crystal) ATR accessory (Specac). The ATR spectrum was obtained, at a resolution of $4 \mathrm{~cm}^{-1}$, 
by adding 500 scans. See Fig.2 for peak assignment of the vibrational modes of the mixed perovskite.

\section{Author's contribution}

M.P. performed the experiments and analyzed the data, S.K. performed the electro-optical measurements, conceived the idea, and made data analysis, T.B. and D.M.B. made PMIRRAS measurements and analyzed the data, S.A. supervised, drafted, and directed the research. All authors contributed to the draft and prepared the final version.

\section{Conflicts of interest}

The authors declare no conflict of interest.

\section{Acknowledgments}

This work received funding from the European Union H2020 Programme under a European Research Council Consolidator grant [MOLEMAT, 726360]. PARASOL, the Spanish Ministry of Science and Innovation.

\section{Supporting information}

The scheme, PM-IRRAS stretching bands, device statistics, steady-state device performance, and admittance spectroscopy.

\section{References}

1. Jung, H. S.; Park, N. G. Perovskite Solar Cells: from Materials to Devices. Small, 2015, 11, 10-25.

2. Grätzel, M. The light and Shade of Perovskite Solar Cells. Nat. Mater., 2014, 13, $838-842$. 
3. Bi, C.; Wang, Q.; Shao, Y.; Yuan, Y.; Xiao, Z.; Huang, J. Non-wetting SurfaceDriven High-Aspect-Ratio Crystalline Grain Growth for Efficient Hybrid Perovskite Solar Cells. Nat. Commun., 2015, 6, 1-7.

4. Mao, L.; Stoumpos, C. C.; Kanatzidis, M. G. Two-dimensional Hybrid Halide Perovskites: Principles and Promises. J. Am. Chem. Soc, 2018, 141, 1171-1190.

5. Smith, I. C.; Hoke, E. T.; Solis- Ibarra, D.; McGehee, M. D.; Karunadasa, H. I. A Layered Hybrid Perovskite Solar- Cell Absorber with Enhanced Moisture Stability. Angew. Chem. Int. Ed., 2014, 126, 11414-11417.

6. Alanazi, A. Q.; Kubicki, D. J.; Prochowicz, D.; Alharbi, E. A.; Bouduban, M. E.; Jahanbakhshi, F.; Mladenović, M.; Milić, J. V.; Giordano, F.; Ren, D. Atomic-Level Microstructure of Efficient Formamidinium-Based Perovskite Solar Cells Stabilized by 5-Ammonium Valeric Acid Iodide Revealed by Multinuclear and Twodimensional Solid-State NMR. J. Am. Chem. Soc, 2019, 141, 17659-17669.

7. $\quad$ Liang, J.; Zhao, P.; Wang, C.; Wang, Y.; Hu, Y.; Zhu, G.; Ma, L.; Liu, J.; Jin, Z. $\mathrm{CsPb}_{0} .{ }_{9} \mathrm{Sn}_{0 .}{ }_{1} \mathrm{IBr}_{2}$ Based All-Inorganic Perovskite Solar Cells with Exceptional Efficiency and Stability. J. Am. Chem. Soc, 2017, 139, 14009-14012.

8. Grancini, G.; Roldán-Carmona, C.; Zimmermann, I.; Mosconi, E.; Lee, X.; Martineau, D.; Narbey, S.; Oswald, F.; De Angelis, F.; Graetzel, M. One-Year Stable Perovskite Solar Cells by 2D/3D Interface Engineering. Nat. Commun., 2017, 8, 1-8. 9. Tsai, H.; Nie, W.; Blancon, J.-C.; Stoumpos, C. C.; Asadpour, R.; Harutyunyan, B.; Neukirch, A. J.; Verduzco, R.; Crochet, J. J.; Tretiak, S. High-Efficiency TwoDimensional Ruddlesden-Popper Perovskite Solar Cells. Nature, 2016, 536, 312-316.

10. Pegu, M.; Haris, M. P.; Kazim, S.; Ahmad, S. Understanding and Harnessing the Potential of Layered Perovskite-Based Absorbers for Solar Cells. Emergent Materials, 2020, 1-28. 
11. Katan, C.; Mercier, N.; Even, J. Quantum and Dielectric Confinement Effects in Lower-Dimensional Hybrid Perovskite Semiconductors. Chem. Rev., 2019, 119, 3140-3192.

12. Liu, S.; Sun, S.; Gan, C. K.; Del Águila, A. G.; Fang, Y.; Xing, J.; Do, T. T. H.; White, T. J.; Li, H.; Huang, W. Manipulating Efficient Light Emission in TwoDimensional Perovskite Crystals by Pressure-Induced Anisotropic Deformation. Sci. $A d v ., 2019,5$, eaav9445.

13. Li, H.; Wang, X.; Zhang, T.; Gong, X.; Sun, Q.; Pan, H.; Shen, Y.; Ahmad, S.; Wang, M. Layered Ruddlesden-Popper Efficient Perovskite Solar Cells with Controlled Quantum and Dielectric Confinement Introduced via Doping. Adv. Funct. Mater., 2019, 29, 1903293.

14. Wang, Z.; Lin, Q.; Chmiel, F. P.; Sakai, N.; Herz, L. M.; Snaith, H. J. Efficient Ambient-Air-Stable Solar Cells with 2D-3D Heterostructured ButylammoniumCaesium-Formamidinium Lead Halide Perovskites. Nat. Energy, 2017, 2, 1-10.

15. Grancini, G.; Nazeeruddin, M. K. Dimensional Tailoring of Hybrid Perovskites for Photovoltaics. Nat. Rev. Mater., 2019, 4, 4-22.

16. García-Benito, I.; Quarti, C.; Queloz, V. I.; Orlandi, S.; Zimmermann, I.; Cavazzini, M.; Lesch, A.; Marras, S.; Beljonne, D.; Pozzi, G. Fashioning Fluorous Organic Spacers for Tunable and Stable Layered Hybrid Perovskites. Chem. Mater., 2018, 30, 8211-8220.

17. Zhou, Q.; Liang, L.; Hu, J.; Cao, B.; Yang, L.; Wu, T.; Li, X.; Zhang, B.; Gao, P. High- Performance Perovskite Solar Cells with Enhanced Environmental Stability Based on a (p- $\left.\mathrm{FC}_{6} \mathrm{H}_{4} \mathrm{C}_{2} \mathrm{H}_{4} \mathrm{NH}_{3}\right)_{2}\left[\mathrm{PbI}_{4}\right]$ Capping Layer. Adv. Energy Mater., 2019, 9, 1802595. 
18. Cho, K. T.; Zhang, Y.; Orlandi, S.; Cavazzini, M.; Zimmermann, I.; Lesch, A.; Tabet, N.; Pozzi, G.; Grancini, G.; Nazeeruddin, M. K. Water-Repellent Low-Dimensional Fluorous Perovskite as Interfacial Coating for 20\% Efficient Solar Cells. Nano Lett., 2018, 18, 5467-5474.

19. Sadhu, S.; Aqueche, K.; Buffeteau, T.; Vincent, J.-M.; Hirsch, L.; Bassani, D. M. Unexpected Surface Interactions between Fluorocarbons and Hybrid Organic Inorganic Perovskites Evidenced by PM-IRRAS and Their Application Towards Tuning the Surface Potential. Mater Horiz., 2019, 6, 192-197.

20. Zhou, Q.; Xiong, Q.; Zhang, Z.; Hu, J.; Lin, F.; Liang, L.; Wu, T.; Wang, X.; Wu, J.; Zhang, B. Fluoroaromatic Cation- Assisted Planar Junction Perovskite Solar Cells with Improved $V_{\mathrm{OC}}$ and Stability: The Role of Fluorination Position. Solar RRL, 2020, 4, 2000107.

21. Li, P.; Dong, H.; Xu, J.; Chen, J.; Jiao, B.; Hou, X.; Li, J.; Wu, Z. Ligand OrientationInduced Lattice Robustness for Highly Efficient and Stable Tin-based Perovskite Solar Cells. ACS Energy Lett., 2020, 5, 2327-2334.

22. Abate, A.; Saliba, M.; Hollman, D. J.; Stranks, S. D.; Wojciechowski, K.; Avolio, R.; Grancini, G.; Petrozza, A.; Snaith, H. J. Supramolecular Halogen Bond Passivation of Organic-Inorganic Halide Perovskite Solar Cells. Nano Lett., 2014, 14, 3247-3254.

23. Salado, M.; Ramos, F. J.; Manzanares, V. M.; Gao, P.; Nazeeruddin, M. K.; Dyson, P. J.; Ahmad, S. Extending the Lifetime of Perovskite Solar Cells Using a Perfluorinated Dopant. ChemSusChem, 2016, 9, 2708-2714.

24. Lee, J. W.; Park, N. G. Chemical Approaches for Stabilizing Perovskite Solar Cells. Adv. Energy Mater., 2020, 10, 1903249. 
25. Jiang, Y.; Yang, S.-C.; Jeangros, Q.; Pisoni, S.; Moser, T.; Buecheler, S.; Tiwari, A. N.; Fu, F. Mitigation of Vacuum and Illumination-Induced Degradation in Perovskite Solar Cells by Structure Engineering. Joule, 2020, 4, 1087-1103.

26. Ning, J.; Zhu, Y.; Hu, Z.; Shi, Y.; Ali, M. U.; He, J.; He, Y.; Yan, F.; Yang, S.; Miao, J. Gaining Insight into the Effect of Organic Interface Layer on Suppressing Ion Migration Induced Interfacial Degradation in Perovskite Solar Cells. Adv Funct Mater., 2020, 30, 2000837.

27. Song, S.; Yang, S. J.; Choi, W.; Lee, H.; Sung, W.; Park, C.; Cho, K. Molecular Engineering of Organic Spacer Cations for Efficient and Stable Formamidinium Perovskite Solar Cell. Adv. Energy Mater., 2020, 10, 2001759.

28. Bouduban, M. E.; Queloz, V. I.; Caselli, V. M.; Cho, K. T.; Kirmani, A. R.; Paek, S.; Roldan-Carmona, C.; Richter, L. J.; Moser, J. E.; Savenije, T. J. Crystal Orientation Drives the Interface Physics at Two/Three-Dimensional Hybrid Perovskites. J. Phys. Chem. Lett., 2019, 10, 5713-5720.

29. Hope, M. A.; Nakamura, T.; Ahlawat, P.; Mishra, A.; Cordova, M.; Jahanbakhshi, F.; Mladenović, M.; Runjhun, R.; Merten, L.; Hinderhofer, A. Nanoscale Phase Segregation in Supramolecular $\pi$-Templating for Hybrid Perovskite Photovoltaics from NMR Crystallography. J. Am. Chem. Soc., 2021, 143, 3, 1529-1538

30. Monyoncho, E. A.; Zamlynny, V.; Woo, T. K.; Baranova, E. A. The Utility of Polarization Modulation Infrared Reflection Absorption Spectroscopy (PM-IRRAS) in Surface and In Situ Studies: New Data Processing and Presentation Approach. Analyst, 2018, 143, 2563-2573.

31. Sadhu, S.; Buffeteau, T.; Sandrez, S.; Hirsch, L.; Bassani, D. M. Observing the Migration of Hydrogen species in Hybrid Perovskite Materials Through D/H isotope Exchange. J. Am. Chem. Soc, 2020, 142, 10431-10437. 
32. Madhavan, V. E.; Zimmermann, I.; Baloch, A. A.; Manekkathodi, A.; Belaidi, A.; Tabet, N.; Nazeeruddin, M. K. CuSCN as Hole Transport Material with 3D/2D Perovskite Solar Cells. ACS Appl. Energy Mater., 2019, 3, 114-121.

33. van Franeker, J. J.; Hendriks, K. H.; Bruijnaers, B. J.; Verhoeven, M. W.; Wienk, M. M.; Janssen, R. A. Monitoring thermal annealing of Perovskite Solar Cells with In Situ Photoluminescence. $\quad$ Adv. Energy Mater., 2017, 7, 1601822.

34. Li, N.; Zhu, Z.; Chueh, C. C.; Liu, H.; Peng, B.; Petrone, A.; Li, X.; Wang, L.; Jen, A. K. Y. Mixed Cation $\mathrm{FA}_{x} \mathrm{PEA}_{1-\mathrm{x}} \mathrm{PbI}_{3}$ with Enhanced Phase and Ambient Stability Toward High- Performance Perovskite Solar Cells. Adv. Energy Mater., 2017, 7, 1601307.

35. Grancini, G.; Marras, S.; Prato, M.; Giannini, C.; Quarti, C.; De Angelis, F.; De Bastiani, M.; Eperon, G. E.; Snaith, H. J.; Manna, L. The Impact of the Crystallization Processes on the Structural and Optical Properties of Hybrid Perovskite Films for Photovoltaics. J. Phys. Chem. Lett., 2014, 5, 3836-3842.

36. Khan, M. T.; Salado, M.; Almohammedi, A.; Kazim, S.; Ahmad, S. Elucidating the Impact of Charge Selective Contact in Halide Perovskite Through Impedance Spectroscopy. Adv. Mater. Interfaces, 2019, 6, 1901193.

37. Abdulrahim, S. M.; Ahmad, Z.; Bahadra, J.; Al-Thani, N. J. Electrochemical Impedance Spectroscopy Analysis of Hole Transporting Material Free Mesoporous and Planar Perovskite Solar Cells. Nanomaterials, 2020, 10, 1635.

38. Wang, J.; Shi, H.; Xu, N.; Zhang, J.; Yuan, Y.; Lei, M.; Wang, L.; Wang, P. Aza [5] helicene Rivals N-Annulated Perylene as $\pi$ - Linker of $D-\pi-D$ Typed Hole- Transporters for Perovskite Solar Cells. Adv Funct Mater. , 2020, 30, 2002114. 
Table of Content

(a)

Parallel
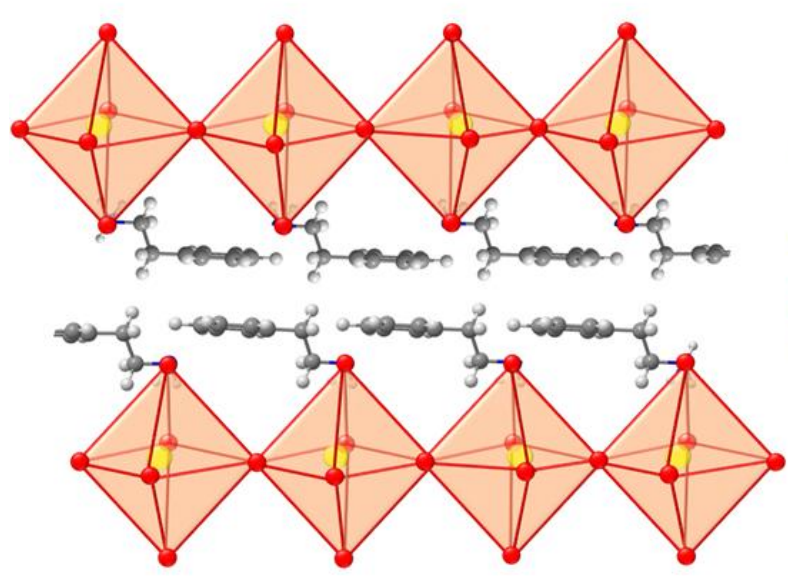

(b)

Perpendicular

$\mathrm{H}$
$\mathrm{F}$
$\mathrm{C}$
$\mathrm{N}$
$\mathrm{I}$
$\mathrm{Pb}$
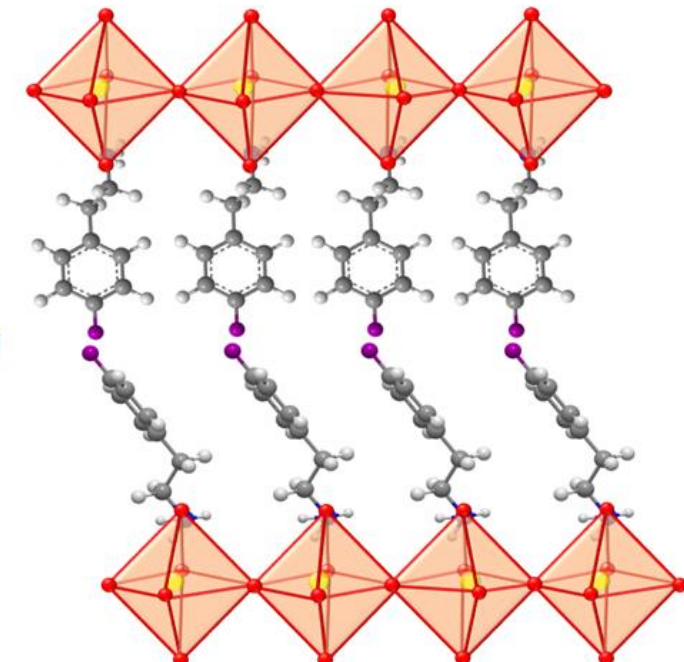